\title{
Espacios de Gobernanza Moderna en Chile: El caso del Consejo Nacional de la Cultura y las Artes
}

\author{
Claudia Baeza \\ Universidad Adventista de Chile
}

\begin{abstract}
Resumen
Esta investigación busca analizar los cambios en la institucionalidad cultural en Chile, del Consejo Nacional de la Cultura y las Artes hacia la creación de un Ministerio de Cultura. Se describe por qué una entidad participativa no tuvo condiciones para responder a las necesidades del sector cultura, siendo necesaria la creación de una institución ministerial. El estudio analiza, desde la perspectiva del diseño de políticas públicas, el rol de los medios de comunicación y de los stakeholders en la discusión sobre la creación del Ministerio de Cultura. Se utilizó la técnica de análisis de contenido cuantitativo, así como se efectuó un análisis documental de fuentes oficiales. Se concluye que la creación de un Ministerio permite develar ante todo el fracaso del Consejo para responder a las necesidades del sector cultural y que la existencia de una figura de tipo bottom up no resistió el peso de la organización político administrativa tradicional. Además, fue posible observar resistencias a la creación del Ministerio, que se relacionan con los contenidos del proyecto, más que con la figura misma.
\end{abstract}

Palabras clave: obernanza, institucionalidad cultural, estrategias emergentes, agenda sistémica, agenda institucional.

Modern Governance spaces in Chile: The case of the National Council for Culture and the Arts

\begin{abstract}
This research analyzes the changes in cultural institutional framework in Chile, from the National Council for Culture and the Arts towards to the creation of a Culture Ministry. It describes why a participative entity was not able to respond to the needs of the cultural sector and was required the creation of a ministerial institution. The study analyzes, from the perspective of public policy design, the media and stakeholders role in the discussion about the creation of the Culture Ministry. The quantitative content analysis technique was used, as well as an official sources documentary analysis was made. It is concluded that the creation of a Ministry can demonstrate first of all the failure of the Council to respond to the needs of the cultural sector and the existence of a bottom-up type figure could not resist the weight of the traditional administrative political organization. Furthermore, it was possible to observe resistance to the creation of the Ministry, which are related to the content of the project, rather than the figure itself.
\end{abstract}

Keywords: Governance, cultural institutional framework, emerging strategies, systemic agenda, institutional agenda.

\section{Espacios de Gobernanza Mo- derna}

En Chile, el proceso de institucionalización de las políticas públicas en cultura se dio en forma paralela al resto de América Latina. A partir de 1990 se produjo un proceso progresivo de carácter parti-

\footnotetext{
*Dirección de correspondencia [Correspondence address]:

Claudia Baeza, Universidad Adventista de Chile

E-mail: claudiabaeza@unach.cl
}

cipativo para la institucionalización de las políticas públicas de cultura. Comenzando por la "Comisión Garretón" en 1991, hasta la creación en julio del 2003 del Consejo Nacional de la Cultura y de las Artes (CNCA), organismo encargado de la institucionalidad política administrativa y de diseñar e implementar las políticas culturales. El Consejo de la Cultura desde sus inicios se ha caracterizado como una institución participativa, donde el diseño de las políticas se realiza a través de un directorio con académicos, intelectuales, políticos entre otros; luego de la realización de Convenciones anuales en las que participan los representantes de las orga- 
nizaciones culturales, además de organizaciones de la sociedad civil. Todo este diseño responde a una organización bottom up, lo que significa que son las bases las que proponen las inquietudes y temas a tratarse en las políticas públicas. En este sentido, el Consejo de la Cultura ha funcionado con un claro énfasis en la participación en el diseño de las políticas culturales, y emerge como un modelo participativo único en el país.

Sin embargo, este modelo participativo, fracasó en su misión, dando paso a la creación del Ministerio de la Cultura y el Patrimonio, una institución ministerial con un diseño tradicional top down. Este estudio busca dar cuenta, desde la voz de sus propios actores, qué pasó con esta institución y buscar las razones de su fracaso. Para tal efecto, se indaga el rol que tuvieron los medios de comunicación en esta discusión sobre el nuevo Ministerio de la Cultura y el Patrimonio, además de las diferentes posturas tanto de oposición activa como de apoyo activo, que presentaron los distintos stakeholders en el campo cultural. De esta forma, se plantea como objetivo de investigación analizar el rol de los medios de comunicación y de los stakeholders en la discusión sobre la creación del Ministerio de Cultura, para de esta forma poder explicar cómo influyó el debate parlamentario en el fin del modelo de institucionalidad participativa.

\section{Gobernanza y Modelo de participa- ción en el diseño de políticas públicas}

Para Renate Mayntz (2002), la gobernanza moderna es una forma participativa de gobernar, en el cual el diseño de las políticas públicas no sólo es definido por las autoridades estatales, sino que además participa la sociedad civil en la formulación e implementación de las políticas públicas. Así, el Estado y las instituciones provenientes de la sociedad civil, cooperan en distintos niveles, a través de negociaciones y redes políticas. Para que esto pueda ocurrir, hay tres pre-requisitos fundamentales: 1) las instituciones deben ser fuertes y competentes y poseer los recursos suficientes realizar sus objetivos; 2) debe haber un mínimo de igualdad en la sociedad civil, en particular igualdad de derechos; y 3) los intereses privados deben poder expresarse de manera organizada (Concepción Montiel, 2006).

\section{Estrategias Emergentes}

El concepto de estrategias emergentes (Mintzberg y Jorgensen, 1995) permite comprender el proceso de formulación de la Política Cultural. En palabras sencillas, la estrategia es lo que la organización realiza. Si la estrategia intencional es realmente ejecutada, esto se denomina 'estrategia deliberada', en cambio si la estrategia ejecutada no era intencional, se trataría de una 'estrategia emergente'. Mientras que la estrategia deliberada corresponde al modelo tradicional y racional de formulación de políticas públicas, en la estrategia emergente "se reconoce que las organizaciones pueden aprender mientras se formulan las estrategias, que pueden responder al resultado de sus propias acciones" (Mintzberg y Jorgensen, 1995:33). Sin embargo, ambas estrategias corresponden a tipos puros, lo que sucede en la práctica es que se presentan ambos tipos de estrategia: una estrategia deliberada puede devenir en una estrategia emergente.

En lo que respecta a las políticas públicas en el Consejo de la Cultura, se puede apreciar que la formulación de éstas, no corresponde a una estructura rígida y de tipo tradicional racional (top down). Esta presenta instancias participativas vinculantes, que han permitido descentralizar y generar participación en una diversidad de actores. De este modo, los espacios participativos produjeron insumos como las líneas estratégicas, programas o proyectos concretos, que influenciaron la definición de las políticas culturales para el quinquenio 2011-2016. Esto permite bajar las decisiones, descentralizándolas.

El concepto de estrategia emergente explica cómo una política se puede transformar en el camino, generar aprendizaje y adaptarse a un nuevo escenario o reformulación de la estrategia. En el contexto de las políticas culturales, estas corresponden a esta forma de hacer política.

\section{Formación de Agenda, Actores y Me- dios de Comunicación Masiva}

La formación de agenda (Agenda-setting) permite comprender el rol que juegan los medios de comunicación en la opinión pública. Se define como "el estudio de los cambios sociales y de la estabilidad social $[. .$.$] , es referirse al conjunto de temas selec-$ cionados para formar parte de un índice o agenda" (Dearing y Rogers 1996, citado por Rodríguez Díaz, 2004:16). Es necesario dar cuenta de la existencia de tres tipos de agenda claramente diferenciados (Rodríguez Díaz, 2004:17).

I La agenda de los medios: su variable principal reside en la medición de un tema cualquiera de los medios de comunicación".

II La agenda pública: mide la importancia que tiene "la selección de determinados temas entre la audiencia o el público". 
III La agenda política: se centra . ${ }^{\text {en las respuestas }}$ y propuestas que ofrecen los grupos políticos y las instituciones sociales sobre determinados temas. Temas que son objeto de debate público y que, en parte, aparecen en las agendas de los medios o en las del público".

La importancia de la agenda de los medios reside en el rol que juega un determinado medio de comunicación en materia de cobertura de un asunto específico, esto es medido de acuerdo a la cantidad de primeras planas, cantidad de páginas, tiempo dedicado, etc., lo anterior determinará la relevancia que le otorgará la audiencia a dicho tema (Rodríguez Díaz, 2004:18). De acuerdo a lo anterior, la importancia que le otorga la audiencia a un tema específico está determinado por la cobertura que realicen los medios, por lo tanto, para llegar a la agenda sistémica es necesario instalar el tema, en primer lugar, en la agenda de los medios. La agenda de los medios influencia en la agenda política, y esta a su vez influye en la agenda de los medios. Mientras que por su parte, la agenda de los medios es el "seleccionador de noticias".

La agenda pública es el grado o jerarquía de importancia que da el público a determinados aspectos noticiosos durante un período de tiempo [...] sobre la dirección de la influencia que ejerce cada una de las agendas, incluido el mundo real [...] la conclusión general de las investigaciones realizadas sobre la agenda-setting es que la agenda de los medios selecciona la agenda del público.

(Dearing y Rogers 1996, Rodríguez Díaz, 2004:17:20-21).

En cuanto, a la agenda política ${ }^{1}$, esta "mide el tipo de acciones que adoptan los gobiernos, parlamentos y las diferentes instituciones sociales, que más tarde formarán parte desencadenante de debates [...] Es la encargada de generar temas nuevos que influenciarán la agenda de los medios y la del público" (Rodríguez Díaz, 2004:22).

Luego de diferenciar los tipos de agenda, es importante determinar cómo se instala un tema en la Agenda. Para que un tema se constituya en parte de la agenda (Cobb y Elder, citado por Parsons, 2007:159), depende de las características del mismo tema para alcanzar a un público más extenso:

a) grado de especificidad: mientras menos específico es el tema puede generar mayor interés público.

\footnotetext{
${ }^{1}$ De acuerdo al planteamiento de Cobb y Elder se denomina agenda institucional.
}

b) alcance de la importancia social.

c) relevancia temporal: a largo plazo genera más interés.

d) grado de complejidad: depende de una definición en términos simples.

e) precedencia categórica: mejor es el caso de carencia de precedentes.

Sumado a lo anterior, los autores Cobb y Elder proponen una diferenciación entre la agenda sistémica y la agenda institucional, mientras que la agenda sistémica es el conjunto de los temas percibidos por los miembros de cierta comunidad política, la agenda institucional corresponde a los temas explícitamente señalados para recibir consideración por parte de los tomadores de decisiones, de forma tal que para que un tema sea parte de la agenda pública, primero debe ser parte de la agenda institucional (Cobb y Elder 1972, citado por Parsons, 2007:158).

\section{El Consejo Nacional de la Cultura y las Artes}

Antes de la creación del CNCA, el diseño de las políticas públicas estaban a cargo de tres ministerios: Ministerio Secretaría General de Gobierno, el Ministerio de Educación y el Ministerio de Relaciones Exteriores. La ejecución de políticas estaba a cargo de varios organismos públicos dependientes de estos ministerios. Por un lado, el sector patrimonial estaba a cargo del Consejo de Monumentos Nacionales de Educación y de la Dirección de Bibliotecas, Archivos y Museos dependientes del Ministerio de Educación; por otro lado, la ejecución de políticas en cultura era función del Departamento de Cultura de la Secretaría de Comunicación y Cultura dependiente del Ministerio Secretaría General de Gobierno, del Comité Calificador de Donaciones Culturales, del Consejo Nacional del Libro, del Fondo Nacional de Desarrollo Cultural y de las Artes, del Consejo de Calificación Cinematográfica dependientes del Ministerio de Educación, y de la Dirección de Asuntos Culturales del Ministerio de Relaciones Exteriores.

En el año 2003, por medio de la ley 19.891, se creó el Consejo de la Cultura y las Artes. Uno de los objetivos de la creación del Consejo de la Cultura fue subsanar la institucionalidad "fragmentada [...] en múltiples organismos gubernamentales de cultura, y dispersa puesto que tales organismos dependían de diferentes ministerios y no existía ninguna instancia de coordinación interministerial de esos organismos." (Squella, 2009:116). El gran consenso 
generado en torno a la creación de este organismo pasa por la historia del país. Durante la década de 1990 la frase "Chile está en deuda con la cultura" era bastante recurrente (Squella, 2009:118). Había una premura entre los "agentes culturales por disponer de una nueva institucionalidad cultural pública, una tarea pensada mas no realizada" (Squella, 2009).

De lo anterior, la primera interrogante que surge es una que se refiere al tipo de institución instaurada, es decir la preferencia por un Consejo y no un Ministerio. Siguiendo a Squella (2009:123), la preferencia por un Consejo se explica por la necesidad de corregir la dispersión y fragmentación de la institucionalidad cultural con una figura (el Consejo), que poseía todas las ventajas de un ministerio, sin ninguno de sus inconvenientes, lo que ahora se ha diluido en el tiempo. Además, el Consejo permitía que el órgano directivo superior interno fuese colegiado y pluripersonal, es más, su presidente al tener rango de Ministro se podría relacionar directamente con el Presidente de la República y acceder a todas las facultades de un Ministro, como asistir a las comisiones del Senado y de la Cámara de Diputados, entre otras (Squella, 2009:124).

Luego de la aprobación de la creación del Consejo, se inició un "período de instalación e implementación del CNCA, [que] se ha desarrollado a través de dos procesos: la conformación de sus órganos constitutivos y la dotación de su infraestructura de funcionamiento" (Moreno, 2013:31). En esta nueva institucionalidad, por un lado, se mantuvo a la Dirección de Bibliotecas Archivos y Museos, el Consejo de Monumentos Nacionales y el Consejo de Calificación Cinematográfica en el Ministerio de Educación. Mientras que por otro lado, se creó la nueva figura del Consejo Nacional de la Cultura y de las Artes para diseñar las políticas públicas bajo un modelo bottom up. Siguiendo a Peters (Moreno, 2013), el proceso de formulación de políticas culturales ha seguido el modelo de tipo bottom up desde sus inicios en el año 2003.

En términos de participación, la formulación de políticas culturales del Consejo de la Cultura, es el resultado de un proceso de consulta participativa; de algún modo, un laboratorio de la gobernanza local. Sin embargo, la institucionalidad de nuestro país es de tipo burocrática y tradicional, por lo que el Consejo en su definición de políticas no escapa a la exclusión de algunos sectores de la sociedad (Moreno, 2013:48-51).

La institucionalidad pública organizada a partir de la creación del Consejo, sufrió grandes cambios debido principalmente a su carácter participativo. Toda la institucionalidad cultural se transformó en torno a este organismo. La conformación del actual Consejo de la Cultura, en materia de organismos públicos, adolece de una debilidad, debido a que los organismos como la Dirección de Bibliotecas Archivos y Museos y el Consejo de Monumentos Nacionales, que representan la institucionalidad pública en materia de Patrimonio, aún permanecen en el Ministerio de Educación.

Las expectativas esperadas; por un lado, sobre la estructura, la participación, las facultades, el resguardo del patrimonio, entre otras, y por otro lado, la falta del alcance y logros de éstas a cabalidad, que con el pasar del tiempo se han hecho más evidentes; generan tensiones dentro del Consejo y entre el Directorio y cada gobierno, y que especialmente se acentuó en el período 2010-2014. Es más, lejos de resolverse, estas tensiones se acentúan, especialmente en la relación entre el Directorio y el Ministro Presidente del Consejo. Este contexto, llevó a la elaboración de un proyecto que sustituye el CNCA y crea el Ministerio de la Cultura y el Patrimonio.

La participación dentro del CNCA es una institución, en el sentido que constituye la esencia del Consejo, plasmada en sus objetivos. En consecuencia, la creación del Ministerio genera una serie de interrogantes sobre el futuro de instancias como la Convención Nacional, los Consejos Sectoriales, los Consejos Regionales, entre otras. La pregunta que se cierne sobre todo el campo cultural es ¿qué sucederá con la participación dentro de una institución como un Ministerio?

\section{Metodología}

El método de investigación que se aplicó fue un Estudio de Caso, debido al proceso inductivo y flexible que permite explorar, describir y explicar. Se realizó un análisis de contenido cuantitativo de prensa y un análisis cualitativo de los documentos de las actas, discursos oficiales, cuentas públicas de la directiva del CNCA, discursos presidenciales y de la discusión parlamentaria sobre institucionalidad cultural y creación del Ministerio de Cultura.

\section{Criterios de selección de las fuentes de información}

En primer lugar, es necesario establecer que se decidió trabajar sólo con prensa escrita, a pesar de la gran proliferación y consumo de prensa on line. Sin embargo, para el presente estudio se determinó que trabajar con prensa on line llevaría a sesgar la recopilación de información, debido a que esta pla- 
taforma es usada principalmente por menores de 30 años, de esta manera "las fronteras entre medios de comunicación de masas y demás formas de comunicación son difusas" (Castells, 2009:100), así el fenómeno de autocomunicación de masas es tan difuso que sería pertinente abordarlo en un estudio a posteriori.

En segundo lugar, para determinar cuáles diarios de prensa escrita analizar, se recurrió al estudio realizado por la Universidad Diego Portales y Feedback publicado bajo el título de "Primer Estudio Nacional sobre Lectoría de Medios Escritos" (2010), considerando como factor inclusivo los medios escritos que presentaron mayor presencia nacional. De acuerdo a la información entregada por el estudio citado, se trabajó con los diarios La Tercera y El Mercurio. Los criterios para determinar la inclusión de éstos, se basó en el posicionamiento del diario, de un total de 8 diarios nacionales, La Tercera es el segundo diario más leído (23\%) a nivel nacional, mientras que El Mercurio es el cuarto más leído $(17 \%)$, sin embargo, ambos medios son los que obtienen mayor promedio en términos de tiempo de dedicación durante el fin de semana, lo que para el estudio fue trascendente, ya que los informes de cambios políticos y legislativos se informan durante los fines de semana. La delimitación del tiempo del estudio corresponde al período desde el 2 de mayo del año 2013 hasta el 30 de abril del 2014 .

La unidad de registro del estudio corresponde a las frases "Ministerio de Cultura"; "ministro de cultura"; "Consejo Nacional de la Cultura y de las Artes"; "proyecto de ley"; y finalmente "RedCultura". Mientras que la unidad de contexto abarcó ambos diarios en su totalidad, se recopilaron las unidades de registro desde el cuerpo principal del diario; la sección de cultura y/o espectáculos; reportajes; especiales y editoriales. Sólo se dejó fuera de la unidad de contexto las secciones de deportes y economía.

Para el análisis cuantitativo se utilizó un sistema de codificación de frecuencias simples, por lo tanto, se puede establecer la preponderancia de una unidad de registro por sobre otra, en la medida que aumenta su frecuencia, en este caso no se utilizaron frecuencias ponderadas debido a que las frecuencias obtenidas no fueron tan altas como para recurrir a este ordenamiento.

Por otro lado, para el análisis cualitativo se establecieron las categorías: participación, trabajo, industrias culturales, institucionalidad. Pero luego, se sumaron las categorías: rol del Estado en cultura, Redcultura, integración, rol de las regiones, internacionalización, educación y cultura, patrimonio y donaciones culturales. Debido a la presencia de éstas en todos los discursos oficiales.

\section{Resultados}

\section{Análisis de actores: la discusión parla- mentaria sobre la creación del Minis- terio de Cultura.}

Del análisis de la discusión de los miembros de la Comisión de Cultura, se observa que había mayormente apoyo de legislar en torno a un Ministerio de Cultura, sin embargo, el apoyo disminuye cuando se hace referencia específicamente al proyecto de ley presentado por el ex-presidente Piñera.

En el campo cultural los diversos actores tomaron sus posturas frente al proyecto de ley que crea el Ministerio de la Cultura y el Patrimonio que se encuentra en el Congreso. De este modo, sus posturas se hicieron escuchar por medio de cartas y documentos a la Comisión de Cultura y las Artes, y especialmente en la Audiencia Pública (29 de julio 2013). Así, a partir de estos registros, se determinó la postura de los actores frente a este proyecto.

De estas intervenciones en la Cámara y en la Comisión de Cultura y las Artes, se ha desarrollado un mapa de actores. Las variables consideradas para los ejes son: el eje X va de "Oposición Activa" a "Apoyo Activo". Mientras que el eje Y va de "Mucha Influencia" a "Poca Influencia".

Los actores del cuadrante A, presentan una oposición activa al proyecto de ley a través de declaraciones en prensa y en la Comisión de Cultura y de las Artes de la Cámara de Diputados, pero tienen poca influencia en el proceso de discusión del proyecto y en su forma definitiva, aquí encontramos instituciones como SIDARTE y SECH, entre otros. El cuadrante B, por otra parte, contiene a los actores que apoyan el proyecto de ley, pero que tienen poca influencia. El único actor identificado es la Cámara Chilena del Libro, la que además no es clara en su apoyo, situándose en el medio entre el apoyo y la oposición. En el cuadrante C, en donde se sitúan los actores con apoyo activo al proyecto y alta influencia en el proceso de promulgación del proyecto de ley, encontramos a los parlamentarios pertenecientes a la Comisión de Cultura y las Artes de la Cámara de Diputados, al Ministro de Cultura y al Presidente de la República. En particular, los parlamentarios que presentaron mayor apoyo al proyecto de ley son los que pertenecen a la misma ala política del gobierno. Por último, en el cuadrante D, en donde se sitúan los actores con alta influencia y oposición al proyecto de ley, en- 
Figura 1: Mapa de actores

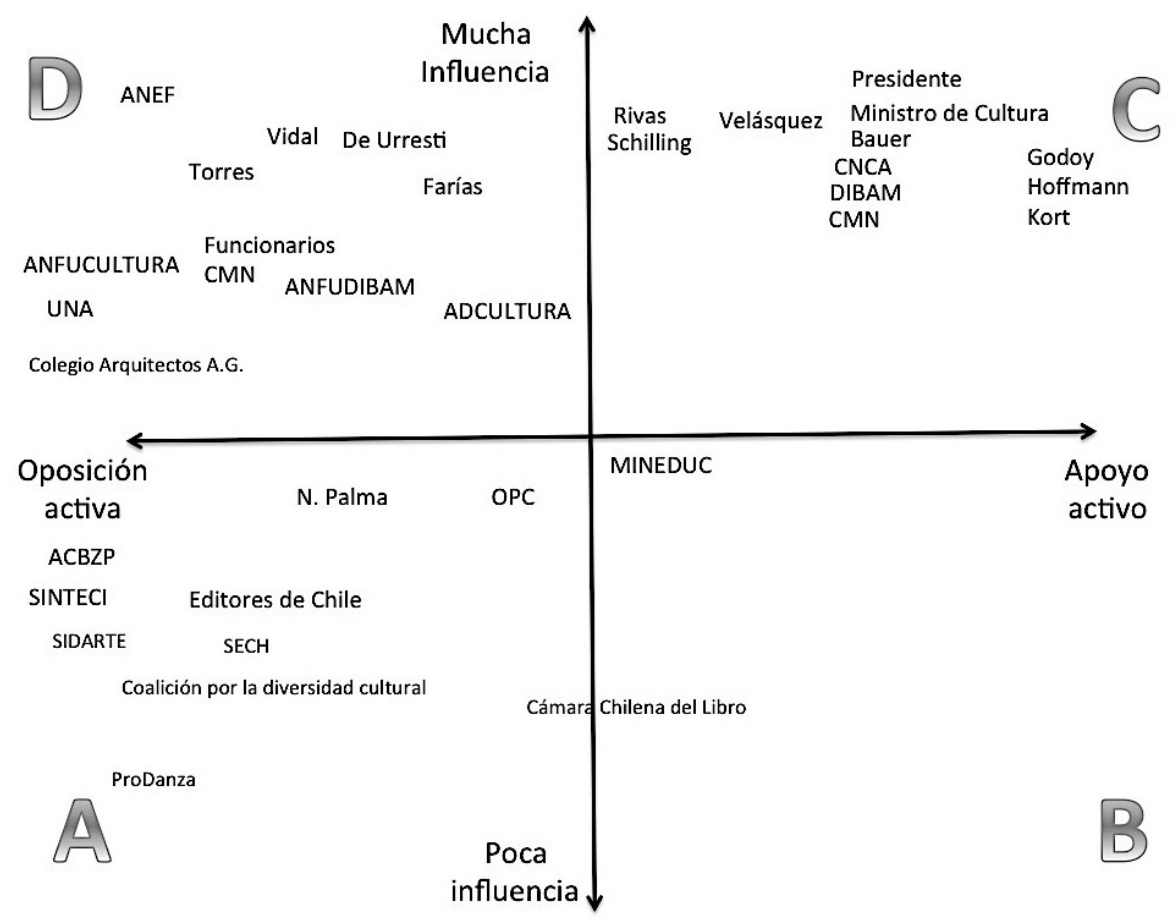

Fuente: modelo tomado de Rietbergen-Mc Craken y Narayan (1998:83 citado por Serra, 2013:72) y aplicación propia a partir de Actas de la Comisión de Cultura de la Cámara de Diputados

(Baeza, 2016)).

contramos a trabajadores de instituciones culturales y a parlamentarios del ala política contraria al gobierno.

Una de las preocupaciones más relevantes planteadas por los actores, fue el tema de la participación. La preocupación se resume en la capacidad que tendrá la nueva institucionalidad cultural para mantener la participación en la formulación de políticas públicas (Ramón Farías, diputado; Manón Herrera, presidenta ANFUCULTURA; Roberto Ampuero, Ministro de Cultura y el Patrimonio).

Un segundo tema relevante tiene que ver con las condiciones laborales en la nueva institucionalidad. Este aspecto se resume en la regularización laboral de los trabajadores de la institución. En particular, quienes levantan esta inquietud son los representantes de Asociaciones de trabajadores públicos del área cultural (Asociación Nacional de Funcionarios de la Dirección de Bibliotecas, Archivos y Museos y Asociación de Funcionarios Consejo de Monumentos Nacionales; Nivia Palma, Miembro del Comité Directivo de la Fundación de la Música; Asociación Nacional de Empleados Fiscales).

Otro tema planteado en la discusión del proyecto de ley, es la posibilidad de integrar a los dis- tintos pueblos que integran Chile; es decir las posibilidades del nuevo Ministerio para reconocer la diversidad cultural del país (Manón Herrera, presidenta ANFUCULTURA; Alan Trumper, director DIBAM).

También se plantean inquietudes con respecto a las Industrias Culturales, en el sentido de que los modelos de negocios, fomentan una lógica de competencia, más no de cooperación ni asociatividad. Se plantean inquietudes con respecto a que los ciudadanos se transformen en meros consumidores de cultura (Rosario Carvajal, presidenta Asociación Chilena de Barrios y Zonas Patrimoniales).

Con respecto a la Institucionalidad Cultural, el principal tema planteado, es la estructura que debe tomar el nuevo Ministerio para agrupar a la dispersión de instituciones que son parte del mundo de la cultura, para así eliminar la duplicidad de funciones (Colegio de Arquitectos de Chile A.G; Edgardo Bruna, Unión Nacional de Artistas; Manón Herrera, presidenta ANFUCULTURA). Además, se plantea que el Ministerio debería llevar a cabo políticas públicas que reúnan a todas las principales instituciones culturales (Roberto Ampuero, Ministro de Cultura y el Patrimonio). Por último, se plantean leyes sectoriales para las distintas artes. 


\section{Análisis de prensa: la discusión en los medios sobre la creación del Ministe- rio de Cultura.}

Durante el análisis de contenido cuantitativo realizado al diario La Tercera, se observó la prevalencia de las palabras "cultura", "cultural", çentro cultural", RedCultura", "Ministerio de Cultura", "Ministro de Culturaz "proyecto de ley" (sobre el ministerio). Tanto çulturaçomo çultural"se mencionan en relación a temas tan variados desde "cultura económica" hasta "cultura ancestral".

La prevalencia del "Ministro de Cultura" en prensa es mayor, ya que sus apariciones no son acotadas sólo al ministerio. Esto se explica principalmente, porque sus apariciones también estuvieron presentes en determinados hechos noticiosos, como lo fue el incendio de edificios patrimoniales, los cambios a la ley sobre Donaciones CulturalesNotas al pie $^{2}$ y otra serie de acontecimientos.

En el diario El Mercurio, se observa el mismo patrón de mayor presencia en prensa del Ministro y menor presencia de proyecto de ley. Sin embargo, las diferencias entre éstos disminuyen. Además, comparativamente hablando, las frecuencias en torno al proyecto de ley, en este medio aumenta su presencia en un $35 \%$, ya que presentó una frecuencia de 19 menciones, en tanto, que en el diario La Tercera, presentó una frecuencia de 14 menciones, a lo largo de todo el período analizado.

Es importante aclarar que, en el diario El Mercurio hay una sección llamada "Políticas Culturales" dentro del Cuerpo E . Artes y Letras". Es en esta sección donde se presentan la mayor parte de los avances políticos en torno a la creación del Ministerio.

Es posible constatar durante este análisis, que no hubo cobertura en la prensa escrita seleccionada, sobre la aprobación de la legislación del proyecto de la creación del Ministerio de la Cultura y el Patrimonio el día 4 de septiembre por la tarde; sin embargo sí lo hubo en la versión online de La Tercera, lo que podría llevar a la conclusión que, por lo menos La Tercera, presenta diferentes contenidos para cada una de sus plataformas de información.

Ambos medios están continuamente informando en materias culturales, además de publicar columnas de opinión, cartas al director e inclusive editoriales al respecto. Sin embargo, hay un hecho que destacar a este respecto: al indagar más a fondo en los artículos de las unidades de contexto seleccionadas en la muestra, se ha constatado que las mencio-

\footnotetext{
${ }^{2}$ Ley Valdés, aprobada por el Senado el 15 de mayo del año 2013
}

nes de El Mercurio corresponden a las declaraciones oficiales del gobierno, esto es, que la información presentada sobre el Ministerio y el proyecto de ley, es sólo la versión oficial, no hay un análisis crítico (sólo las cartas al director) en torno al proyecto, tampoco un reportaje en profundidad sobre el tema, a pesar de presentar una sección denominada "políticas culturales". Por otro lado, en el diario La Tercera la tendencia de presentar la versión oficial se mantiene, sin embargo, en casos contados se presentan otras visiones al respecto.

\section{Análisis de las declaraciones oficiales sobre la creación del Ministerio de Cultura}

\section{Rol del Estado en la Cultura}

Desde la figura del Ministro de Cultura, el rol del Estado se comprende como acción acotada, regulada y legitimada socialmente:

\begin{abstract}
La promoción de la cultura no puede ni debe identificarse con un sector social o político, porque cada uno de nosotros, sin exclusiones de ninguna naturaleza, tenemos un rol que cumplir en la promoción, circulación o goce de las artes y la cultura [...] La libertad artística y cultural la garantiza justamente la existencia de la diversidad de quienes contribuyen a sostenerla
\end{abstract}

(La Tercera, 17 enero 2014, Roberto Ampuero Ministro de Cultura).

En referencia al rol del Estado, el gobierno actual hace referencia a un concepto más amplio de cultura, a "una cultura del desarrollo y la democracia, que se exprese en la tolerancia, el cultivo de la memoria histórica, el gusto por la innovación" (M. Bachelet, 21 de mayo 2014)

\section{Nueva institucionalidad}

La nueva institucionalidad ha recibido un respaldo explícito como compromiso gubernamental en el período de Bachelet, derivado de su programa de gobierno, como una medida programática que es prioridad de política pública en cultura.

Sin embargo, pese a este compromiso de generar una institucionalidad, se reconocen los avances del período anterior y se establece una innovación, como es la generación de una indicación al proyecto de Piñera, que surge de un proceso participativo, 
recogiendo las demandas de los artistas, mundo patrimonial y sus organizaciones:

\begin{abstract}
Consideraremos la opinión de tres sectores por diferentes mecanismos: los profesionales del mundo de las artes, los trabajadores del Estado en materia cultural y los ciudadanos. La descentralización es clave en este proceso, por lo mismo, los directores regionales de Cultura se entrevistarán con los profesionales de cada una de las regiones. Además, me reuniré con los trabajadores del CNCA, DIBAM y funcionarios del Consejo de Monumentos para escucharlos. Finalmente, abriremos un foro en internet con 22 preguntas sobre el contenido de la ley para recibir desde comentarios hasta propuestas más integrales
\end{abstract}

(La Tercera, 12 Abril 2014, Claudia Barattini Ministra de Cultura).

Una de las indicaciones sustitutivas al primer proyecto de ley, consiste en la creación de un Consejo del Patrimonio paralelo al Consejo de la Cultura ya existente. A través de la prensa se puede dar cuenta que esta indicación estaba entre las prioridades de la nueva cartera, ya que en abril a poco más de 1 mes de asumir, ya se concebía esta idea. Detrás de este planteamiento se encuentra la eliminación de duplicidades, aún existentes en la presente institucionalidad cultural, lo que es una preocupación que cruzó en forma transversal a todo el espectro político.

Según el proyecto del año 2013, se mantendrían los órganos colegiados, y a través de éstos la participación vinculante, sin embargo, no todos los actores culturales están de acuerdo con el tipo de participación por este medio, ya que las bases no logran participar. Se ha mencionado el tema de la autonomía e independencia del Consejo de la Cultura de los gobiernos de turno, especialmente porque su política cultural está diseñada con un horizonte de 5 años (la duración del período presidencial actual es de 4 años) y por el hecho de que es diseñada con participación vinculante de órganos colegiados y un Directorio. No obstante, de algunos sectores existen aprehensiones con respecto a la nueva institucionalidad:
La iniciativa [...] encierra el riesgo de que en la nueva institucionalidad prevalezcan visiones que respondan a los intereses de ciertos grupos de presión, lo que podría conducir a que la política cultural adquiera un sesgo dirigista y que se aleje de un justo reflejo de las distintas sensibilidades y expresiones creativas que surgen en la sociedad [...] resulta indispensable que el nuevo consejo mantenga una pluralidad de visiones y una voluntad de amplio criterio, lo que debería garantizar que las políticas culturales no terminen siendo capturadas por grupos de interés.

(La Tercera, 10 de mayo 2013, Editorial).

\section{RedCultura}

Este tema tuvo bastante presencia tanto en prensa, como en los discursos oficiales de las autoridades involucradas, se trata de un proyecto a largo plazo y de gran envergadura. Partió de una iniciativa anunciada el año 2009 por la presidenta Bachelet y tuvo continuidad en el gobierno siguiente hasta la actualidad,

Si en 2010 había tres espacios inaugurados, al final de esta administración dejaremos 33 construidos o en obras y otros 16 con sus procesos iniciados (sobre la RedCultura) [...] el presupuesto 2014 del CNCA tuvo un incremento del $8,2 \%$ respecto del 2013 [...] Hay una continuidad en la promoción de la cultura y es necesario tener un política cultural de Estado, no de gobierno ni del partido de turno.

(El Mercurio, 18 diciembre 2013, Roberto Ampuero Ministro de Cultura).

Esta declaración reconoce la existencia de los inicios del proyecto, el primer gobierno de Bachelet alcanzó a entregar 3 centros culturales, pero de gran envergadura, entre estos el Centro Cultural Gabriela Mistral.

\section{Participación}

Este es un tema central en la institucionalidad cultural, la mayor preocupación es sobre el futuro de la participación en una estructura ministerial. Por otro lado, la participación también se considera para discutir y retroalimentar el proyecto del Ministerio con los actores involucrados.

En el proyecto del año 2013, la participación permanece en el Consejo de la Cultura y el Patrimonio, tal como se explicó anteriormente, en el anteproyecto sustitutivo se presentan dos consejos, uno de la 
Cultura y las Artes y otro, en forma paralela, el Consejo del Patrimonio. En el primer proyecto de ley, se presenta sólo un Consejo que reúne ambas áreas. En el corazón de este Consejo Nacional de la Cultura y el Patrimonio, se conservaría los órganos colegiados para definir las políticas públicas.

Por último, sobre la profesionalización y compromiso del Directorio, se establece una dieta para sus miembros, ya que una de las fallas del Consejo, era precisamente la falta de profesionalización y compromiso de los integrantes del Directorio, al tener a sus miembros ad honorem, y considerando que cada Director realiza una actividad remunerada aparte, se traduce en la práctica en la dificultad de obtener quórum para realizar las sesiones.

\section{Integración}

En este apartado, se incluye a las regiones, diversidad, multiculturalidad y descentralización. Se pudo apreciar que para los dos últimos Ministros Presidentes Ampuero y Barattini (que corresponden al período que abarcó el estudio) del Consejo es de importancia considerar estas temáticas.

Además, para la Ministra Barattini es de importancia entregar acceso a artistas que no se consideran de consumo cultural masivo, como artesanos y los nuevos creadores.

\section{Donaciones Culturales y Patrimonio}

La nueva ley de donaciones culturales es uno de los avances más anunciados por el gobierno de Piñera, consistió en ampliar el universo de posibles donantes y beneficiarios, "se perfeccionarán diversos aspectos de la actual legislación, ampliando positivamente la gama de potenciales donantes y beneficiarios, reduciendo las restricciones innecesarias y simplificando los procedimientos" (El Mercurio, 20 de mayo 2013, Editorial). Además, es de gran importancia en lo que se refiere a cuidados y protección del patrimonio.

Desde el Ministerio, las declaraciones en materia de donaciones se centran en llamar a la ciudadanía a participar en la promoción cultural, particularmente luego de la entrada en vigencia (01.01.2014) de la ley 20.675, que modifica la ley Valdés (artículo $8^{\underline{0}}$ de la ley $\left.\mathrm{N}^{\mathrm{o}} 18.985\right)$.

Por último, los temas: internacionalización, educación y cultura, no fueron recogidos en las declaraciones en prensa, pero sí es posible apreciar estos temas a cabalidad en los discursos oficiales de los Presidentes de la República y Ministros Presidente del Consejo.

\section{Análisis de Cuentas Públicas Anuales: Presidencial y Ministerial}

El discurso del año 2014 es coherente con las declaraciones de los actores culturales, sobre la fragmentación de la institucionalidad cultural, y en consecuencia, la necesidad de una institución mayor que reúna a todas las instituciones culturales. El llamado a un Ministerio "más participativo" por parte del ministro Ampuero, se concreta con la cartera de la ministra Barattini, ya que se llama a una consulta participativa, incluyendo una consulta a pueblos originarios, para socializar y generar nuevas aristas para el proyecto sustitutivo que se presentará este año.

Se puede observar la continuidad del programa RedCultura por más de 5 años, lo que se traduce en más de un período presidencial. Este tiene sus inicios en el año 2009 con la construcción del Centro Cultural Gabriela Mistral como el primero de una red de Centros Culturales. Se trata de una política pública exitosa, que en su primera fase de implementación, se cumplió y se ha ido avanzando en el tiempo; de un centro cultural, se ha extendido a una red de más de treinta centros. Según la última Cuenta Pública, se está trabajando en una segunda fase que contemplaría la provisión de personal para estos centros.

Para el gobierno anterior, la Ley de Donaciones Culturales se convirtió en su caballo de batalla, un logro para la administración, ya que esta ley se encontraba entre las prioridades de la administración Piñera. Sin embargo, hubo algunas desconfianzas al respecto cuando se discutía, especialmente sobre el hecho de ampliar el universo de donantes. Finalmente, permitirá un avance en materia patrimonial debido a que se abre la posibilidad de beneficiar a dueños de inmuebles patrimoniales. Se observa que ambos ministros consideran la ley donaciones culturales como un gran avance en cultura. En la última Cuenta Pública la ministra Barattini menciona capacitaciones en torno a la ley, lo que permite socializarla, de modo de que se genere más participación en materia de donaciones.

La categoría internacionalización de la cultura se incluyó en el análisis, debido a que ambos ministros hacen alusión al tema de la internacionalización en sus respectivas cuentas. Importante es el apoyo a los artistas a través de programas. De este modo, en esta materia se aprecia un consenso en relación a la meta a alcanzar, visibilizar a los artistas nacionales en el exterior y, parafraseando al ministro Ampuero, fortalecer la identidad chilena en el ex- 
tranjero.

El tema de la integración es de gran importancia, debido a que Chile es un país muy diverso. Por lo tanto, toda política pública naciente de una institución, debe considerar la inclusión de las diversas realidades existentes en el país. De este modo, se incluyen los diversos orígenes tanto étnicos, sociales, económicos y territoriales. El respeto por el Convenio 169 , por medio de la consulta indígena, es un gesto político de la actual administración que se circunscribe en esa línea. Asimismo, ambos Ministros en sus Cuentas Públicas reconocen la existencia de una identidad diversa, y además Barattini plantea la necesidad de "la implementación de políticas culturales que promuevan, desde un enfoque de derechos, la difusión y conocimiento de las culturas de los pueblos originarios".

Con respecto a la participación, se deslinda, por un lado, que esta consiste en tomar voz (consultiva) y decisión (vinculante) en los procesos de toma de decisiones atinentes al Consejo; y por otro lado, en el acceso a la expresión y consumo artístico cultural. Para el primer caso, es necesario la existencia de organizaciones empoderadas con capital social para poder participar en el campo cultural, mientras que en el segundo caso, el Estado se comporta como garante de derecho a la cultura y a desarrollar creatividad y expresión cultural. En relación, a la participación dentro del Consejo y el futuro Ministerio, aún hay mucho que revisar para establecer una determinada orientación, aunque las consultas ciudadanas aplicadas muestran una señal, en pos de la participación.

La relación entre educación y cultura siempre ha sido muy estrecha, hay que considerar que hace pocos años atrás los temas de cultura, correspondían a una división del Ministerio de Educación. En materia de cultura, se han implementado algunos programas para generar interés y enseñar diversas disciplinas culturales, lo central es que son llevadas a cabo por medio de artistas y expertos del área correspondiente, programas como Acciona y la Red Nacional de Centros de Servicios Culturales trabajan esa línea. Además, el fomento a la lectura desde la edad temprana anunciado por la Presidenta Bachelet, lo que no deja de ser destacable, considerando el déficit en materia de horas/libro y comprensión lectora que detentan los estudiantes chilenos ${ }^{3}$.

Sobre la presencia de las regiones, se trata de una debilidad institucionalidad antigua. Esta debilidad, cruza todo la administración pública, no es sólo una

\footnotetext{
${ }^{3}$ De acuerdo, a los resultados del estudio PISA (Programme for international student assessment) para el año 2012, el promedio de los estudiantes chilenos con 441 puntos obtenidos en el test, se ubica por debajo del promedio (496) de los países pertenecientes a OECD (2012).
}

característica del Consejo de la Cultura. El ministro Ampuero al mantener su residencia en Valparaíso, hace un gesto simbólico por visibilizar más esa región. Sin embargo, se trata de una acción que permanece en el simbolismo, ya que la mayoría de la planta ya sea a contrata u honorarios del Consejo permanece en Santiago, por mencionar un ejemplo. El realizar las Convenciones y entregar los Premios en Cultura en regiones distintas a la Metropolitana, es otro gesto en esta línea, debido a que lo central permanece en Santiago. Este tema también se cruza con la participación, porque de lo que se trata en relación a las regiones, es sobre la participación de éstas en diversas esferas, tanto como en la decisión como en la organización de eventos culturales que perduren en el tiempo.

La declaración del ex-ministro Ampuero en relación al tema dice . ${ }^{\mathrm{a}}$ mpliar los espacios de decisión de las propias regiones sobre el rumbo a seguir en su expansión cultural, incluyendo la definición del destino de los recursos". Es por decirlo, de algún modo, demasiado progresista, ya que en el tipo de administración actual las instituciones regionales están limitadas en materia decisional. El Consejo, en este sentido, ha presentado avances en comparación con el resto de la institucionalidad pública, por medio de los Consejeros regionales quienes realizan una serie de decisiones que otros organismos públicos no ejercen. No obstante, las falencias en esta materia se espera sean subsanadas en la nueva estructura institucional, con la dieta que percibirán los Consejeros, y con la posibilidad de postular a concursos públicos en cultura.

Para el ex-ministro Ampuero, el rol de Estado se basa en promover el respeto en la diversidad, además de generar la tan deseada descentralización y regionalización del país, de manera de visibilizar las diferencias. Para el gobierno de Bachelet el Estado debe ser garante, en tanto que la cultura es vista como un derecho consagrado en la Declaración de universal de los derechos humanos, por lo que al Estado, le corresponde "garantizar este derecho y democratizar el acceso a la cultura, estimular la creación artística, proteger el patrimonio, promover la diversidad de expresiones culturales, asegurar una justa retribución por el trabajo creativo y reposicionar la educación artística". Para lograr esta meta, se plantea al Estado más que un supervisor del campo, sino como un hacedor, un actor central en el campo de la cultura.

El mundo del patrimonio, se presenta como un legado histórico, la historia contada a través de personas, inmuebles, costumbres, expresiones culturales, etc. Es por esta razón, que se habla de patrimonio material e inmaterial. Además, este tema se considera importante debido a la necesidad de rescatar 
la memoria. Finalmente, la existencia del Ministerio reunirá al área patrimonial y cultural en una sola estructura, de modo de mejorar la gestión en esta área.

\section{Conclusiones}

\section{Análisis de medios}

La prensa es un actor central en la fijación de los temas en la agenda pública. A través del análisis a los medios, se pudo observar que los temas de cultura muchas veces son invisibilizados en los medios. El proyecto de ley que crea el Ministerio del ramo se trata de un hito muy importante, sin embargo ningún medio escrito cubrió esta noticia.

La agenda de los medios es fijada por un actor con los medios de producción para producir noticias. Tal como se pudo observar, los medios cumplen el rol de seleccionador de noticias, en tanto que el público se informa (conformando la agenda pública) de acuerdo a los contenidos ya establecidos por los mass media. Tanto la agenda de los medios como la agenda política se influencian entre sí (Rodríguez Díaz, 2004). Para el caso específico de la creación del Ministerio de Cultura (que ahora deviene en Ministerio de las Culturas, las Artes y el Patrimonio) ha sido la agenda de los medios, quien ha fijado el tema de la cultura en la agenda sistémica.

El tema de la cultura en los medios es cubierto de manera tal, que no logra resaltar en la agenda de los medios, y por lo tanto, tampoco en la agenda pública. Es un tema presente, pero que no logra la importancia de temas como salud, economía o educación. Aunque, se pudo observar presencia en ambos medios de prensa, durante el período de recopilación de datos, hubo meses sin menciones del Ministro de Cultura, del Ministerio y del Proyecto. Por lo tanto, de acuerdo a la teoría de formación de agenda (Rodríguez Díaz, 2004), el tema del Ministerio no es de prioridad ni se encuentra instalado en la agenda pública, debido a la baja cobertura de los medios de este tema. Siguiendo a Cobb y Elder (citado por Parsons, 2007), es posible afirmar que el tema ha llegado a la agenda institucional de acuerdo a la instalación de este tema en la agenda sistémica. Son los actores con participación quiénes han resituado el tema en la agenda. Es un tema que vuelve a reflotar, luego que el Consejo haya dejado insatisfecho a gran parte del campo cultural, lo que fue posible evidenciar en las declaraciones de los actores.

Por otra parte, se reconoce la falta de apoyo de algunos sectores de la cultura a algunos contenidos del proyecto, más no a la creación de un Ministerio. Por lo tanto, lo que se encontraba en cuestionamiento no era el hecho de crear una institución de tipo ministerial, sino que ciertos contenidos específicos del proyecto en sí.

\section{Razones del fracaso del modelo parti- cipativo}

A partir al análisis de la discusión en torno al proyecto de ley que consagra el Ministerio de la Cultura y el Patrimonio, es posible detectar varias causas que permiten explicar el fracaso del modelo participativo del Consejo Nacional de la Cultura y las Artes.

Específicamente, lo que se refiere a gobernanza moderna se puede observar en el trabajo de formulación de las políticas culturales, ya que las políticas culturales son el resultado de un proceso participativo vinculante, que incluye a representantes del campo cultural y autoridades del mismo. De este modo, las estrategias emergentes (Mintzberg y Jorgensen, 1995) en políticas, programas o proyectos es el modus operandi, que mejor refleja la forma de hacer políticas públicas en el sector cultural, ya que fue necesario formular y reformular, enfrentar escenarios inesperados o no considerados en la formulación de políticas al momento de implementarlas.

En consecuencia, a la luz de la teoría de gobernanza (Mayntz, 2002), se evidencian falencias en el área de la participación del Consejo. Siguiendo con los pre-requisitos propuestos: si evaluamos el primero, fue posible encontrar casos de políticas culturales que no fueron implementadas por falta de presupuesto o competencia en sus campos específicos. Es decir, políticas que no pudieron efectuarse debido a que el Consejo no tenía las competencias como institucionalidad, o las competencias yacían en un Ministerio diferente al Ministerio de Educación.

El segundo pre-requisito para tener gobernanza moderna plantea que es fundamental la existencia de "una sociedad civil fuerte, funcionalmente diferenciada y bien organizada [...] Una sociedad civil no puede existir donde hay gran desigualdad social" (Mayntz, 2002). Al revisar esta condición, hay dos hechos centrales que destacar: en primer lugar, en Chile no existe una sociedad civil fuerte, por varias razones que tienen relación con su historia política y social. En segundo lugar, el país presenta una enorme desigualdad social. Es decir, sólo por no cumplir esta condición, una gobernanza real y participativa, debería comenzar por cambios en la sociedad en su conjunto, una sociedad que presente equidad so- 
cial y económica, lo que permitirá entregar el marco para tener gobernanza moderna.

Finalmente, la última condición que plantea Mayntz tiene relación con las características necesarias dentro de una sociedad civil muy organizada, sin embargo, en el caso de Chile presenta una enorme carencia. Si bien es cierto que durante las últimas décadas se han creado una enorme cantidad (existen por lo menos 55 organizaciones no gubernamentales) de ONG's, como representantes de la sociedad civil en las más variadas materias, estas no tienen el poder político, ni presentan el nivel de organización necesaria para la gobernanza moderna. Esto se suma a la falta de actores corporativos en el país.

Lo anterior es trascendental para el caso estudiado, ya que muchas de las limitaciones tienen que ver con este contexto. Se trata de un campo en el cual no fue posible desarrollar la gobernanza moderna, ya que el país carece de las condiciones para que se diera en forma efectiva. Junto con estas carencias, la forma de gobernar en el aparato estatal responde a la burocracia tradicional tipo top down, lo que generaba una piedra de tope para las acciones del Consejo.

De esta manera, se plantea que el Modelo de Institucionalidad Participativa implementado en el CNCA, fracasó por variadas razones.

La primera razón detectada es una pérdida de apoyo de los actores que fomentaron el surgimiento de una institucionalidad como el CNCA. Tal y como fue planteado por Squella (2009), el CNCA tenía la ventaja de tener un carácter ministerial, sin las trabas burocráticas de este. Lo que en la práctica demostró no ser el mejor modelo (Baeza, 2016). "Se evidencia primero la existencia de fuertes resistencias administrativas para hacer efectiva [la] participación. No basta con tener diversos mecanismos participativos, estos no son suficientes si no están acompañados de la voluntad política de implementarlos" (Moreno, 2013:128).

En segundo lugar, fue posible observar que los cambios ocurridos en la estructura jerárquica del CNCA, que pusieron al Directorio a la izquierda del Ministro, generaron tensiones entre estos dos actores. Esto queda ilustrado en el hecho que las tensiones se provocan no en cuanto a la creación del Ministerio, sino que en el modus operandis del gobierno y del Ministro Presidente (Baeza, 2016).

En tercer lugar, sumado a los dos puntos anteriores, y de acuerdo a la Constitución (Moreno, 2013), los Ministros son colaboradores del Presidente de la República, lo que no fue visto con buenos ojos por el Directorio, ya que el cambio realizado el 2009 li- mitaba su accionar como Directorio y le entregaba más atribuciones al Ministro, lo que llevó al Consejo a enfrentar precisamente los deberes de un Ministerio, que querían evitar al crear el Consejo de la Cultura.

Cuarto, por diferencias con los funcionarios: la mayoría de los trabajadores del Consejo de la Cultura trabajan a honorarios, lo que significa que carecen de estabilidad laboral y seguridad social. Esto se suma al hacinamiento en las oficinas de Santiago y la falta de uso del edificio principal del Consejo en Valparaíso.

Por último, se observa que el Consejo, al pertenecer al Ministerio de Educación, no tuvo la libertad de acción para implementar parte de sus políticas. Muchas veces, las decisiones tomadas en las instancias participativas, morían en el papel, ya que las decisiones finales eran ministeriales (Baeza, 2016).

\section{Referencias}

Baeza, C. (2016). Trayectorias de la institucionalidad en cultura: del modelo participativo a la creación del Ministerio (2013-2015). Observatorio Cultural.

Castells, M. (2009). Comunicación y poder. Alianza, Madrid.

Concepción Montiel, L. E. (2006). Globalización, democracia y gobernanza global: perspectiva latinoamericana. Politika: Revista de Ciencias Sociales $=$ Gizarte Zientzien Aldizkaria, (2):69-89.

Mayntz, R. (2002). Los Estados nacionales y la gobernanza global. Revista del CLAD. Reforma y Democracia, 24:29-44.

Mintzberg, H. y Jorgensen, J. (1995). Una estrategia emergente para la política públia. Gestión y política pública, IV(1).

Moreno, P. (2013). Participación en la formulación de políticas culturales. Tesis para optar al grado de magíster, Universidad de Chile.

Parsons, W. (2007). Políticas públicas: una introducción a la teoría y la práctica del análisis de políticas públicas. Flacso, México.

Portales-Feedback, U. D. (2010). Primer Estudio Nacional sobre Lectoría de Medios Escritos. Ediciones UDP, Santiago.

Rodríguez Díaz, R. (2004). Teoría de la AgendaSetting: aplicación a la enseñanza universitaria. Observatorio Europeo de Tendencias Sociales. 
Serra, I. (2013). Análisis del proceso de elaboración y aprobación del PRMS 100. Tesis para optar al grado de magíster, Universidad de Chile. tural de Chile. En Robaina, G., editor, Seminario Internacional sobre Institucionalidad Cultural en el Uruguay, pp. 83-148, Montevideo.

Squella, A. (2009). La nueva institucionalidad cul- 